\title{
BMJ open Why do clinicians not refer patients to online decision support tools? Interviews with front line clinics in the NHS
}

\author{
Glyn Elwyn, Andrew Rix, Tom Holt, Deborah Jones
}

To cite: Elwyn G, Rix A Holt T, et al. Why do clinicians not refer patients to online decision support tools? Interviews with front line clinics in the NHS. BMJ Open 2012;2:e001530. doi:10.1136/ bmjopen-2012-001530

\section{- Prepublication history for this paper are available online. To view these files please visit the journal online (http://dx.doi.org/10.1136/ bmjopen-2012-001530).}

Received 22 May 2012 Revised 20 October 2012 Accepted 22 October 2012

This final article is available for use under the terms of the Creative Commons Attribution Non-Commercial 2.0 Licence; see http://bmjopen.bmj.com

Cochrane Institute of Primary Care and Public Health, Cardiff University, Cardiff, UK

\section{Correspondence to}

Professor Glyn Elwyn; elwyng@cardiff.ac.uk, glynelwyn@gmail.com

\section{ABSTRACT}

Objective: To assess whether clinical teams would direct patients to use web-based patient decision support interventions (DESIs) and whether patients would use them.

Design: Retrospective semistructured interviews and web server log analysis.

Participants and settings: 57 NHS professionals (nurses, doctors and others) in orthopaedic, antenatal, breast, urology clinics and in primary care practices across 22 NHS sites given access to DESIs hosted on the NHS Direct website.

Results: Fewer than expected patients were directed to use the web tools. The most significant obstacles to referral to the tools were the attitudes of clinicians and clinical teams. Technical problems contributed to the problems but the low uptake was mainly explained by clinicians' limited understanding of how patient DESIs could be helpful in clinical pathways, their perception that 'shared decision-making' was already commonplace and that, in their view, some patients are resistant to being involved in treatment decisions. External factors, such as efficiency targets and 'best practice' recommendations were also cited being significant barriers. Clinicians did not feel the need to refer patients to use decision support tools, web-based or not, and, as a result, felt no requirement to change existing practice routines. Uptake is highest when clinicians set expectations that these tools are integral to practice and embed their use into clinical pathways.

Conclusions: Existing evidence of patient benefit and the free availability of patient DESIs via the web are not sufficient drivers to achieve routine use. Health professionals were not motivated to refer patients to these interventions. Clinicians will not use these interventions simply because they are made available, despite good evidence of benefit to patients. These attitudes are deep seated and will not be modified by solely developing web-based interventions: a broader strategy will be required to embed DESIs into routine practice.

\section{INTRODUCTION}

Are clinical teams willing to ask patients to use decision support interventions, when hosted on the web? A decade of research has

\section{ARTICLE SUMMARY}

Article focus

- Research studies demonstrate that engaging patients in the use of decision support (decision aids) is beneficial but there are significant challenges to their implementation into practice.

- Clinicians are in a position to advocate the use of patient decision support but do not appear to do so.

\section{Key messages}

- Reluctance to refer patients to decision support is largely based on the scepticism of professionals that these tools add value, coupled with difficulties of incorporating the tools into existing workflows and competing organisational pressures, such as targets.

- This reluctance will not be overcome simply by placing tools on the web.

- When appropriately directed, a significant number of patients do use the decision support and say they find them useful.

Strengths and limitations of this study

- The evaluation study upon which these results are based set out to explore patterns of usage but turned to examine why take up was lower than expected.

- The evidence comes mainly from 57 qualitative interviews with healthcare professionals taking part in the introduction of six web-based decision support tools hosted on the NHS Direct website.

demonstrated consistent positive outcomes when patient decision support interventions, often called decision aids, are evaluated in randomised controlled trials. ${ }^{1}$ Use of these interventions is advocated in order to help achieve shared decision-making-where patients and clinicians work together to arrive at decisions that best match the informed patient preference. ${ }^{2}$ These interventions typically consider clinical situations where there are reasonable alternatives. Typical examples are whether to proceed to 
a total knee replacement or use alternative treatments or whether to accept or decline an amniocentesis, a procedure that runs the risk of leading to a miscarriage. When these interventions are used, patient knowledge increases, risk perceptions are more accurate, patients feel more actively involved in decisions and often tend to make more conservative choices, especially when considering discretionary surgery. ${ }^{1}$

Despite good evidence and increasing policy support, studies have revealed resistance to the implementation of shared decision-making and decision support. ${ }^{3}$ Professionals often hold the view that they 'already do' shared decision-making, that the interventions promoted lack applicability to individual patients and that there is insufficient time to involve patients in decisions. ${ }^{4}$ To date, only a few studies have addressed the use of implementing these interventions in routine clinical settings. ${ }^{5}$ Many obstacles have been described and it is too early to say whether the benefits observed in randomised trials can be replicated. ${ }^{6}$ As yet, there has been no large-scale study to assess the uptake possible in pragmatic settings. ${ }^{7}$

Responding to the potential benefits of shared decision-making and the use of patient decision support, the NHS in England invested approximately $£ 1.5 \mathrm{M}$ in 2009-2010 creating a platform of web-based patient decision support, as part of an England-wide shared decision-making programme. NHS Direct was commissioned by the East of England Strategic Health Authority to adapt, host and pilot the introduction of web-based decision support interventions into the NHS, as part of the Quality Innovation, Performance and Prevention Programme.

A multiphase programme of work was proposed, with the aim of creating an easily accessed web-based set of patient decision support tools hosted on an NHS webplatform coupled with telephone support. ${ }^{2}$ An external evaluation was set up to assess whether clinical teams would direct patients to these tools and to assess uptake and use. This article reports on the attitudes of participating staff which lie behind the reluctance to refer patients to these tools.

\section{METHODS}

Two phases were planned. In each phase, three web interventions were adapted and hosted on NHS Direct's website. NHS sites were recruited into evaluation pilots of approximately 3-month's duration (see table 1).

In phase 1 , the three interventions were adaptations of programmes originally developed by the Foundation for Informed Decision Making, Boston (see table 1). The prostate web interventions were originally produced in DVD versions and had been previously introduced into some clinics in England. ${ }^{89}$

Eight NHS clinics, across England, some with previous involvement in the development of the DVD and webbased versions, agreed to introduce patients to them. For the knee condition, patients referred to secondary care were identified by staff and directed to access the web-based tools to read information about a range of treatment options, for example, physiotherapy, injections and knee replacement. If patients completed the use of the web tool, a personalised summary was generated that listed their preferred treatment. Patients were asked to either print this summary or, when they attended their next clinic appointment, to ask for the summary to be discussed with them. It had been estimated that 360 patients across three clinics would be eligible to use the knee osteoarthritis web intervention during the evaluation period. Similar methods were applied to the web tools for benign prostatic hyperplasia (BPH) and localised prostate cancer (LPC).

In phase 2, three interventions were adapted from tools developed at Cardiff University (table 1). Data were collected from seven breast cancer and seven antenatal outpatient clinics recruited by NHS Direct (table 2). Estimates of patients potentially eligible for these tools had been set at 50 per site, a pool of around 700 patients. Primary Care Trusts in England were asked to contact primary care practices, alerting clinicians to the availability of the prostatespecific antigen (PSA) decision support tool.

\section{DATA COLLECTION}

Data were collected in each phase for a maximum of 14 weeks using the following methods:

Table 1 Web-based patient decision support hosted on NHS Direct

Reasonable options considered

Phase 1

Osteoarthritis of the knee (sites 1, 2 and 3)

Benign prostatic hyperplasia (sites 4, 5, 6, 7 and 8) Localised prostate cancer (sites 4, 5, 6, 7 and 8)

Phase 2

Amniocentesis (based on AmnioDex) (16)

Breast Cancer (based on BresDex) (17)

Test for prostate-specific antigen (PSA) (based on

ProsDex) (12)
Pain management, lifestyle change, manual therapy, joint injections, knee replacement

Active monitoring, surgical approaches, medication Active monitoring, surgical approaches, medication

No test, amniocentesis, chorionic villus sampling Breast conservation surgery (lumpectomy) or mastectomy No test, PSA test 2010 February-

Evaluation period

June-August April 2011 
Table 2 Patients use of the web-tools during phases 1 and 2

\begin{tabular}{|c|c|c|c|}
\hline & $\begin{array}{l}\text { Number of patients } \\
\text { directed }\end{array}$ & $\begin{array}{l}\text { Number of patients } \\
\text { accessing }\end{array}$ & $\begin{array}{l}\text { Number using web tool } \\
\text { for }>30 \mathrm{~min}\end{array}$ \\
\hline \multicolumn{4}{|l|}{ Clinics in phase $1^{*}$} \\
\hline Osteoarthritis of the knee (sites 1,2 and 3) & 162 & $102(63 \%)$ & $26(16 \%)$ \\
\hline $\begin{array}{l}\text { Urology: benign prostatic hyperplasia (sites } \\
4,5,6,7 \text { and } 8 \text { ) }\end{array}$ & Not known & 8 & 2 \\
\hline $\begin{array}{l}\text { Urology: localised prostate cancer (sites 4, } \\
5,6,7 \text { and } 8 \text { ) }\end{array}$ & Not known & 24 & 5 \\
\hline \multicolumn{4}{|l|}{ Clinics in phase 2} \\
\hline 1 Amniocentesis & 32 & $20(63 \%)$ & $11(55 \%)$ \\
\hline 2 Amniocentesis & 25 & $3(12 \%)$ & $1(33 \%)$ \\
\hline 3 Amniocentesis & 25 & $3(12 \%)$ & $1(33 \%)$ \\
\hline 4 Amniocentesis & 32 & $9(28 \%)$ & $4(44 \%)$ \\
\hline 5 Amniocentesis & 20 & $3(15 \%)$ & $1(33 \%)$ \\
\hline 6 Amniocentesis & 10 & $6(60 \%)$ & $3(50 \%)$ \\
\hline 7 Amniocentesis & 13 & $10(77 \%)$ & $5(50 \%)$ \\
\hline All antenatal clinics & 157 & $54 / 157$ (34\%) & $26 / 54(48 \%)$ \\
\hline 1 Breast cancer & 12 & $1(8 \%)$ & $1(100 \%)$ \\
\hline 2 Breast cancer & 20 & $0(0 \%)$ & Not applicable \\
\hline 3 Breast cancer & 2 & $0(0 \%)$ & Not applicable \\
\hline 4 Breast cancer & 2 & $0(0 \%)$ & Not applicable \\
\hline 5 Breast cancer & Unknown & 2 & $2(100 \%)$ \\
\hline 6 Breast cancer & Unknown & 0 & Not applicable \\
\hline 7 Breast cancer & Unknown & 1 & $0(0 \%)$ \\
\hline All breast cancer clinics & 36 & 4/36 (11\%) & $3 / 4(75 \%)$ \\
\hline
\end{tabular}

Referral data: In both phases, clinics kept a record of patients directed to use the tools. Patients were given unique access codes, enabling anonymised tracking of server web logs.

Web logs: Web server log data were collected, tracking access (content and duration) of each web page viewed.

Interviews with health professionals: After informed consent was obtained, semistructured interviews were conducted with medical and nursing specialists responsible for implementing the intervention at each clinic. The respondents were asked their views about the webbased patient decision support and whether they had referred patients to them, we asked about the role they might play in their work. Interviews were conducted at the start, mid-point and end of the pilots, audiorecorded and transcribed. Results from the evaluation of the first phase led to a change of focus for the evaluation of the second phase.

Data analysis: Web server log data were verified, cleaned and analysed by TH. AR and DJ coded the interviews independently, meeting to agree coding frames, prior to jointly categorising the data into themes related to clinician views about the decision support tools and their willingness to direct patients to them.

\section{RESULTS}

Patient's access and use: Records indicate that 162 of the estimated potential pool of 360 patients were offered access to the osteoarthritis knee tool: 102 of the $162(63 \%)$ visited the site, at least briefly. A total of $38(23 \%)$ provided some information; 27 patients $(26 \%)$ used the site in sufficiently to produce a summary sheet that could be used in a future clinic appointment. This number represents $7 \%$ of those estimated to have been eligible. No estimates were available for eligible patients in relation to $\mathrm{BPH}$ and $\mathrm{LPC}$, or for numbers directed to the sites. Web log data showed 24 and 8 unique visitors to the LPC and BPH sites, respectively, resulting in 5 and 2 patients using the sites from introductory pages to summary page, answering all questions.

To compensate for the unexpected access rates in phase 1 , the number of participating clinics planned for phase 2 was increased. On the basis of attendance rates, 700 patients had been considered eligible (50 at each of 14 clinics). The actual uptake was less than expected: 157 patients were directed to the amniocentesis web tool and 36 patients were directed to the breast cancer tool (data were only available from four of the seven breast clinics; table 2). Data about the number of patients directed to the PSA website from primary care were not available.

Table 2 also describes considerable variation between clinics in the number of patients who accessed the tool. Three of the antenatal clinics had access rates of $60 \%$ or higher; all the other antenatal clinics had access rates of $28 \%$ or lower. Having gained access, 26/54 (48\%) patients viewed the amniocentesis site for $30 \mathrm{~min}$ or 


\section{Table 3 Interviews undertaken for each intervention}

\section{Number healthcare professionals interviewed}

\begin{tabular}{lr}
\hline Intervention & \\
Osteoarthritis of the knee & 6 \\
Benign prostatic hyperplasia & 4 \\
Localised prostate cancer & 5 \\
Amniocentesis & 19 \\
Breast cancer & 23 \\
Prostate-specific antigen & 0 \\
Total & 57 \\
\hline
\end{tabular}

more, indicating significant levels of interest after overcoming the access hurdle. These data suggest that high proportion of patients will potentially access and use decision support tools: how best to motivate professionals to direct patients to these interventions is a challenge.

Interviews with health professionals: During phases 1 and 2, 57 interviews were conducted with healthcare professionals, across all sites (see table 3). Interviews in phase 2 focused on examining reasons for the unexpected low number of patients directed to use the decision support tools and what steps might be helpful in embedding patient decision support into routine practice. Analysis of the interviews revealed overall resistance to direct patients to use decision support. This resistance arose from existing professional attitudes and to barriers arising from current organisational routines. Table 4 illustrates the main themes found in the data.

\section{Theme 1: Limited motivation to use tools designed} to support patients participate in decisions

The majority of key professionals, specialists, nurses and managers, had been invited to brief introductions about the websites and had been sent an information pack. However, interviews revealed that many clinicians had not fully understood the intended role of the tools. Ideally, decision support tools should be used by patients when diagnosis and treatment options are confirmed. In urology, this is after urine flow dynamics or biopsies have provided a diagnosis; in knee clinics, this is after osteoarthritis has been confirmed as the likely cause of pain. However, many clinicians saw these tools as merely means for providing information and not as ways of engaging patients in discussions about treatment decisions. Clinicians reported being happy with: “... anything that makes the patient genuinely more knowledgeable ..." (orthopaedic surgeon, site 3, phase 1). However, the concept that patients should use these tools to engage in decisions was not widely understood: "Having a summary sheet is fine for the patient but don't expect me to use it ... it would interfere with what I do, although I can see it might lead to less questionswhich is good" (orthopaedic surgeon, site 2, phase 1).

\section{Theme 2: 'We already do shared decision-making'}

The interviews revealed a widely held belief that decisions were already shared with patients and that this was occurring without the need for patient decision support. As one nurse commented, "I don't know how much more they could be involved (in decisions)" (antenatal screening nurse, clinic 7). An antenatal specialist stated that: "... we moved away from the patriarchal ideal of telling people what to do 20 years ago" (specialist, clinic 7). Many clinicians espoused the view that: “... you wouldn't replace the time you spend with patients by giving them a decision tool ... and the shared decision making process ... isn't dependent on having this kind of tool" (breast care nurse, clinic 4).

\section{Theme 3: Perceived patient barriers to involvement in decision-making}

Patients' inability to access the Internet was often raised. "You wonder if they've got access, especially to broadband..." (specialist nurse, site 4, phase 1) and that "This generation are not used to getting information in

Table 4 Summary of themes derived from the qualitative analysis

Theme
1. Limited motivation to use tools designed to support
patients participate in decisions

2. 'We already do shared decision-making'

3. Perceived patients' barriers to involvement in decision-making

4. Organisational factors that reduce professionals' motivation to involve patients in decision-making

\section{Summary}

Low motivation for the intended role of patient decision was encountered, as illustrated by uncertain deployment of the tools in clinical pathways and low uptake by patients

Strong perception that clinicians were already involving patients in decisions, therefore no perceived need to change or to adopt decision support by adapting pathways

Clinicians cited barriers such as technical access problems and often saw patients as those that did not want to be involved in shared decision-making or as those they felt had already accessed information on the internet

External efficiency targets and health professionals' views about the imperative of using effective treatments were significant barriers to introducing patient decision support tools 
this way" (specialist nurse, site 6, phase 1). Clinicians also gave examples of patients resisting involvement in decisions. A surgeon reported one woman saying, "I'll do whatever you say. You tell me to have a mastectomy, I'll have a mastectomy ..." (breast surgeon, clinic 4). A breast care nurse commented, “... most women... say 'you make the decision for me ... it's much easier if you tell me what I have to have done" (breast care nurse, clinic 4). Patients were often categorised as either "sufficiently informed' or misinformed, 'clutching internet printouts' (breast surgeon, clinic 3). The prevailing view among interviewees was to accept, that, "there is a role for your website but that cohort is very narrow..." (breast surgeon, clinic 1).

\section{Theme 4: Organisational factors that reduce professionals' motivation to involve patients in decision-making}

External targets were often cited as barriers. Meeting efficiency targets, particularly in cancer, led to constant pressures. As a specialist explained, the constant pressure to 'get to a decision' quickly means that clinicians do not "... want to encourage indecisive patients go off (to read a web tool), and then ... come back and see us for another consultation" (breast surgeon, clinic 1).

Many clinicians were explicit that they would not direct patients to tools that do not support their own views about effective treatments. For example, the breast cancer tool lists mastectomy and breast conservation surgery as treatments that should both be given consideration by women who have early breast cancer. Yet, many clinics have a different ethos. As one surgeon stated "We obviously try to do breast conservation whenever we can. That's the basic premise..." (breast surgeon, clinic 1). This attitude is supported by external audits, where high mastectomy rates are viewed as inappropriate.

Routines were perceived as already being under pressure and so asking "... a midwife to give (information about a web tool) was yet another thing ..." (antenatal screening coordinator, clinic 1). Existing processes were viewed as being satisfactory: "I would say that what we're doing is actually fine" (screening midwife, clinic 4) and that the website's “information does not make women's decision about amniocentesis any easier" (specialist, clinic 7). In short, it was felt that sufficient information was already available: most clinics did not see the websites as adding value to their work, and therefore saw no need for change. Nevertheless, three antenatal clinics achieved relatively high uptake rates, more importantly perhaps, access rates of $60 \%$ or higher (clinics 1, 6 and 7; see table 2). Interviews revealed that these three clinics had specifically modified their pathways and were alerting women by telephone about the tool at the same time as giving them their 'high risk' result and offer of amniocentesis. Women at clinic 7 were encouraged to access the website before attending their amniocentesis counselling session. Where the tools are integrated into routines, and expectations set that patients will use them before making decisions, different patterns of use emerge.

\section{DISCUSSION}

Principal findings: This evaluation demonstrates that the implementation of patient decision support into routine practice is unlikely to be accomplished by the development of web-based tools alone: the relatively low uptake of the tools was explained by clinicians' uncertainty about the content and also where exactly to position them in their clinical pathways, their perception that 'shared decision-making' was already commonplace in their teams. A minority also felt that patients, on the whole, are resistant to engaging in decisions. In addition, there was evidence that external factors, such as efficiency targets and 'best practice' recommendations, are viewed as have more influence in driving decisions than the informed preferences of patients.

In short, clinicians did not feel the need to direct patients to use decision support tools, web-based or not, and, as a result, felt no requirement to change existing routines. Taken together, these views represent significant barriers and explain why, in most of the clinics, few patients were directed to use the tools.

It was encouraging, however, to notice that the data revealed that many patients, when directed to these tools, are willing to use them. Uptake is highest when clinicians set expectations that these tools are integral to practice and embed their use into clinical pathways.

Strengths and weakness of methods: The strengths of the study are the pragmatic use of routine NHS settings, the purposive sampling of those who had been actually tasked to introduce patients to these web-based decision support tools. The use of web server logs to track access provides direct data about the actual use of the tools. Constraints were placed on the intensity of the evaluation processes by the tight service delivery timetables set by the NHS sponsor.

Although relevant clinicians were interviewed in all sites, the sample could have been expanded if more time had been available. Although the study sheds some light on some the implementation barriers, further work is needed into understand how to identify patients that would benefit from being asked to use decision support, that is, to determine the eligible numbers in any given clinical pathway as a way of establishing the denominator population for assessing uptake.

Results in context of other similar work: Previous work on web-based patient decision support interventions has been typically undertaken in the context of randomised trials $^{1}$ and so it is difficult to draw comparisons to this pragmatic implementation study. Nevertheless, existing research studies do illustrate high degrees of difficulty in recruiting patients to use web-based interventions, even with dedicated resources and incentives. ${ }^{10-12}$ Holmes Rovner documented a decade ago that engaging clinicians to direct patients to use decision support tools active participants was a significant barrier to implementation. ${ }^{13}$ Caldon also documented of the concerns and anxieties of health professionals about patient decision support, such as the threat to clinical autonomy, the fear 
of more demanding patients and suspicion that the content was did not accord with their personal practice. ${ }^{4}$ These findings are echoed by recent reviews. ${ }^{6} 1415$

Implications: As recently noted by Coulter, ${ }^{7}$ addressing the implementation challenge will require efforts targeted at changing hearts, minds and systems. More preparation and training might be helpful but future work will also need to work on achieving organisation-wide agreement about the role of these interventions and their location in clinical pathways.

Acknowledgements We acknowledge the support and advice of the following people. Marie Anne Durand, at the time Programme Manager of the UK SDM Programme, Steven Laitner, SDM Programme Lead, Nick Chapman, Chief Executive Officer NHS Direct and Shirley Large, Head of Research NHS Direct.

Contributors GE and AR conceived the idea of the study and were responsible for the design of the study. AR and DJ collected the data and, with the addition of TH and GE, analysed the data. The initial draft of the manuscript was prepared by GE and AR and then circulated repeatedly among all authors for critical revision. GE is the guarantor.

Funding NHS Direct were commissioned by the East of England Strategic Health Authority to adapt, host and evaluate the use of patient decision support in England. NHS Direct provided funding for the evaluation, considered the evaluation under their research governance process and given the service evaluation nature of the study, decided that ethical approval was not required. NHS Direct had no involvement in the drafting of this article.

Competing interests None.

Provenance and peer review Not commissioned; externally peer reviewed.

Data sharing statement There are no additional data available.

\section{REFERENCES}

1. Stacey D, Bennett C, Barry M, et al. Decision aids for people facing health treatment or screening decisions. Cochrane Database Syst Rev 2011:(10);CD001431.
2. Elwyn G, Coulter A, Laitner S, et al. Implementing shared decision making in the NHS. BMJ 2010;341:c5146.

3. Légaré $\mathrm{F}$, Ratté $\mathrm{S}$, Gravel K, et al. Barriers and facilitators to implementing shared decision-making in clinical practice: update of a systematic review of health professionals' perceptions. Patient Educ Couns 2008;73:526-35.

4. Caldon L, Collins K, Reed M, et al. Clinicians' concerns about decision support interventions for patients facing breast cancer surgery options: understanding the challenge of implementing shared decision-making. Health Expect 2010;14:133-46, Blackwell Publishing Ltd.

5. Feibelmann S, Yang TS, Uzogara EE, et al. What does it take to have sustained use of decision aids? A programme evaluation for the Breast Cancer Initiative. Health Expect 2011; 14(Suppl 1):85-95.

6. Légaré $\mathrm{F}$, Ratté $\mathrm{S}$, Stacey $\mathrm{D}$, et al. Interventions for improving the adoption of shared decision making by healthcare professionals. Cochrane Database Syst Rev 2010:(5);CD006732.

7. Coulter A. Engaging patients in healthcare. New York: Open University Press, 2011.

8. Wirmann E, Askham J. Implementing patient decision aids in urology. Oxford: Picker Institute. 2006.

9. Garden J. Implementing an informed decision making programme for urology patients. $J$ Commun Healthcare 2008;1:297-310.

10. Frosch DL, Bhatnagar V, Tally S, et al. Internet patient decision support a randomized controlled trial comparing alternative approaches for men considering prostate cancer screening. Arch Intern Med (Internet) 2008;168:363-9.

11. Hirsch $\mathrm{O}$, Keller $\mathrm{H}$, Krones $\mathrm{T}$, et al. Acceptance of shared decision making with reference to an electronic library of decision aids (arriba-lib) and its association to decision making in patients. An evaluation study. Implement Sci (Internet) 2011;6:70.

12. Evans R, Elwyn G, Edward A, et al. A randomised controlled trial of the effects of a web-based PSA decision aid, Prosdex. Protocol. BMC Fam Pract 2007;8:58.

13. Holmes-Rovner MV, Valade D, Orlowski C, et al. Implementing shared decision-making in routine practice: Barriers and opportunities. Health Expect 2000;3:182-91, Blackwell Science Ltd.

14. Gravel K, Légaré F, Graham I. Barriers and facilitators to implementing shared decision-making in clinical practice: a systematic review of health professionals' perceptions. Implement Sci 2006;1:16.

15. Elwyn G, Scholl I, Tietbohl C, et al. The implementation of patient decision support interventions into routine clinical practice: a systematic review. Implement Sci 2012; In review. 